\title{
Ginseng extract and its constituents alleviate cisplatin toxicity and reverse cisplatin resistance
}

\author{
Jian Cai ${ }^{1}$, Qiulie Zhang ${ }^{2}$ and Huihong Huang ${ }^{3 *}$ \\ ${ }^{1}$ Department of Pharmacy, Fengxian Mental Health Center, Fengxian, China \\ ${ }^{2}$ Department of Pharmacy, Jinhai Community Health Center, Fengxian, Shanghai, China \\ ${ }^{3}$ Department of Pharmacy, Fengxian Traditional Chinese Medicine Hospital, China
}

\begin{abstract}
Cisplatin (DDP) is one of most used anticancer agents. It has shown antitumor activity in testicular cancer, head and neck cancer, ovarian cancer, lung cancer, malignant lymphoma and bladder cancer. It causes much toxicity such as skin and mucous membranes toxicity, ototoxicity and nephrotoxicity especially at higher dose, which might be involved with DNA damage and subsequently renal cell death. Ginseng is a group of cosmopolitan plants. As the root of Panax ginseng C.A. Meyer, ginseng is well known as a tonic medicine for restoring and enhancing human health. Ginseng extract contains numerous phytochemicals such as ginsenoside, phenols and acidic polysaccharides that have significant pharmacological activities. In vivo and in vitro studies demonstrated that ginseng extract and its constituents are able to prevent cisplatin-induced toxicity through reducing the oxidative stress by restoring the antioxidant enzymes level and exerting anti-inflammatory effect. Multidrug resistance (MDR) is a principal obstacle to successful cancer chemotherapy. Because of low toxicity and diversity of effect, ginseng extract constituents have been attached more and more attention in MDR reversal. Evidences showed that ginseng extract and its constituents are promising potential for cisplatin resistance in cancer treatment.
\end{abstract}

\section{Introduction}

Cisplatin (cis-diammine-dichloro-platinum II, DDP) is an important anticancer drug. Peyrone first discovered Cisplatin in 1844 and Werner resolved its chemical structure in 1893. Unfortunately, cisplatin did not gain much attention until 1965 when Rosenberg discovered it inhibited bacterial cell division. The miraculous discovery followed an observation that an electric current delivered to bacterial culture via platinum electrodes led to inhibition of bacterial growth. The active compound was cisplatin, which demonstrated subsequently to exert major activity against several tumors in mice.

Later in 1969, Rosenberg demonstrated cisplatin possessed antitumor activity in a mouse model. In 1979, cisplatin obtained approval by the Food and Drug Administration for the treatment of solid tumors [1]. For a few decades cisplatin is a widely used as part of drug combination that can cure testicular cancer and, in combination with other drugs, for palliation of a variety of solid tumors. It causes little toxicity to bone marrow by itself but can add to the toxic effects of other drugs and may lead to anemia. Its major dose-limiting toxicities are severe nausea and vomiting, damage to the kidneys, and loss of hearing and neurotoxicity after prolonged use. The toxic effects on the kidneys may be reduced by maintaining a rapid urine output during and after drug administration $[2,3]$.

Currently, in the field of cancer chemotherapy greater global interest arises in non-synthetic, natural products derived from plant sources due to better tolerance and minimum adverse drug reactions as compared to synthetic medicines. The patients with certain chronic medical conditions, including cancer, liver disease, human immunodeficiency, asthma and others disorders especially prefer to use natural products [4]. As an important source of drugs, natural products play an important role in the discovery and development of new drugs.
In fact, more than $60 \%$ of anti-tumor drugs are closely related to natural products [5]. A few natural products are effective in the supportive cancer care in neo-adjuvant and adjuvant chemotherapy [6]. From a global perspective, the search for effective adjuvant chemotherapy from natural products has never stopped [7].

In order to prevent cisplatin high levels of toxicity, many researches are conducted to explore potential natural products protective effects both in animal experiments and in clinical practice [8]. Natural products are multifarious chemical substances produced by living organisms. They usually include multiple components that have a multiple targets and multiple pharmacological effects. Up to now, natural products with broad medicinal properties have been studied extensively to reduce severe anticancer drugs toxicity. Meanwhile these natural products have also been studied to reverse resistance of anticancer drugs. Natural products that exert both prevent toxicity of anticancer drugs and reverse MDR are particularly noteworthy our attention. In this review, we focus on ginseng extract on cisplatin toxicity prevention and resistance reversal. We hoped that by analyzing the different aspects of the source, mechanism of action and biological activity in vitro and in vivo, we would find new members that can be applied in clinical cancer chemotherapy in the near future.

${ }^{*}$ Correspondence to: Huihong Huang, Fengxian Traditional Chinese Medicine Hospital, 9588 Nanfeng Hwy, Shanghai 201400, China, E-mail: HYPERLINK "mailto:1142269435@qq.com"1142269435@qq.com

Key words: ginseng extract, chemical constituent, cisplatin, toxicity, resistance, review

Received: November 23, 2019; Accepted: December 13, 2019; Published: December 16, 2019 


\section{Action and toxicity of cisplatin}

Cisplatin acts by a mechanism that is similar to that of classical alkylating agents. The chlorine atoms may be displaced directly by nucleophilic groups of DNA or indirectly after chloride ions are replaced by hydroxyl groups through reaction of the drug with water. The preferred sites for binding of cisplatin to DNA are the N-7 positions of guanine and adenine base. Cisplatin binds to two sites on DNA with the production of interstrand cross-links and interstrand adducts. Lethal effects against cancer cells seem to correlate with the number of cross-links formed between the DNA strands. These cross-links continued to form for several hours after removal of the drug and then repaired.

Following administration, cisplatin is bound to plasma proteins rapidly and tightly, with greater than $90 \%$ of free cisplatin lost in the first $2 \mathrm{~h}$. Total cisplatin (free and bound drug) disappears more slowly from plasma, with a prolonged half-life of 2 to 3 days. Cisplatin is excreted mainly via the urine, and 15 to 30 percent of the administered dose is excreted during the first $24 \mathrm{~h}$.

Cisplatin may cause much toxicity such as skin and mucous membranes toxicity, gastrointestinal tract reaction, bone marrow suppression, peripheral neuritis, ototoxicity, and nephrotoxicity especially at higher dose, which restricts its clinical application [2,3]. About $70 \%$ of cancer patients develop acute kidney injury (AKI) with cisplatin treatment [9], which results in a negative impact on longterm renal function and patient survival. In fact, cisplatin-induced nephrotoxicity is the main reason to discontinue cisplatin chemotherapy in $17 \%$ of patients.

Cisplatin-induced nephrotoxicity usually occur as early as 8 to 15 $\mathrm{h}$ following high dose of cisplatin $(45 \mathrm{mg} / \mathrm{kg})$ injection as evidenced by high blood urea nitrogen (BUN). Renal injury occurs mainly in the proximal, distal convoluted tubule and collecting duct. The organic cation transporter-2 and copper transporter-1, which are expressed in the basolateral membrane of proximal tubular cells, mediate the cellular transport of cisplatin into the renal cells [10]. Then cisplatin taken up from the basolateral compartment is transported to and accumulated in the apical compartment of the proximal tubular cells over time. Cisplatin concentration in the proximal tubular cells is approximately 5 times higher than that in the serum [11]. In the presence of the lower salt concentration, cisplatin is then converted into a highly reactive molecule, producing cis- $\left[\mathrm{Pt}(\mathrm{NH} 3)_{2} \mathrm{Cl}(\mathrm{H} 2 \mathrm{O})\right]^{+}$ [12] . The positive charge on the platinum complex can be attracted to the negatively charged nuclear DNA, and thus interferes with DNA replication and/or transcription, causing DNA damage [11]. The DNA damage and associated DNA damage response (DDR) are important pathogenic mechanism of AKI, where severe DNA damage often leads to renal cell death. DDR pathway of ATR/Chk2/p53 is activated in cisplatin-induced nephrotoxicity and thus leads to renal tubular cell apoptosis [11]. Cisplatin affects the microscopic structure of the kidney characterized by apoptosis and necrosis of proximal tubules, formation of abundant tubular protein casts, large foci of inflammatory cells and interstitial fibrosis.

Oxidative stress is also implicated for cisplatin-induced nephrotoxicity [13]. Following cisplatin administration, it induces the production of $\mathrm{H}_{2} \mathrm{O}_{2}$ and $8-\mathrm{OHdG}$ (renal oxidative stress product 8-hydroxy-2'-deoxyguanosine) which is significantly associated with decreased GSH (glutathione) levels, and serum creatinine (SCr) level and creatinine clearance reductions. Cisplatin-induced oxidative stress activates the p38MAPK pathway, which lead to caspase-3 expression.
Cisplatin also induces lipid peroxidation and decreases gluconeogenesis in the kidney cortex, which displayed to affect pathways in proximal tubular cells, i.e. p53 signaling, Nrf2 mediated oxidative stress response, mitochondrial processes, mTOR, AMPK signaling, actin nucleation via the ARP/WASP complex and regulation of cell polarization.

In general, cisplatin causes nephrotoxicity usually via inhibiting cisplatin efflux in renal tubular endothelial cells, inducing cell apoptosis and inflammatory reaction.

\section{Drug resistance of cisplatin}

Many types of cancer in humans (e.g., colon cancer, lung cancer) have a relatively low probability of response to treatment with anticancer drugs. Other human tumors (e.g., breast cancer, ovarian cancer) often respond to initial treatment, but acquired resistance to further therapy usually prevents drug treatment from being curative. Many factors may influence the resistance to chemotherapy. Cisplatin is no exception. Resistance to cisplatin may develop by multiple mechanisms.

Cisplatin causes cellular damage by binding with DNA, leading to cross-linkages and breaks in DNA strands. Cells may be resistant to cisplatin through a number of general mechanisms, including decreased cellular uptake (increased lung-resistant protein, LRP), inactivation by binding to sulfhydryl (e.g., increased conjugation by GSH), increased DAN repair (increased removal of cisplatin-DNA adducts) and decreased ability to undergo apoptosis.

Natural products as well as their derivatives share common mechanisms of resistance. Many are substrates of membrane-based proteins that act to pump the drugs out of cells. The best characterized is P-glycoprotein (P-gp), which is encoded by the multiple drug-resistance (mdr) genes. $\mathrm{P}$-gp is known to be one of a family of molecules, referred to ATP-binding cassette $(\mathrm{ABC})$ transporter proteins molecules. This family includes multidrug resistance associated protein (MRP), whose expression causes resistance to multiple anticancer drugs. LRP is also associated with resistance to multiple drugs and probably causes export of drugs from cells. Alternative mechanisms include changes in the activity of the enzyme topoisomerase II, changes in GSH and other sulfhydryls, and DNA repair enhancement [15].

\section{Ginseng alleviate cisplatin toxicity}

Panax ginseng C.A.Meyer is the roots of perennial herbaceous plant, which belongs to Panax genus under Araliaceae family. According to the processing method, ginseng can be made into red ginseng and black ginseng. Red ginseng is made of fresh ginseng by soaking, cleaning, sorting, steaming, drying and processing, which is usually produced in Korea and known as Korean red ginseng (KRG). Furthermore, fermented black ginseng (FBG) is made of red ginseng by heating repeatedly at high temperature, also known as nine steaming and nine processing $^{15}$. In general, as a well-known tonic medicine for restoring and enhancing human health in traditional Chinese medicine history, ginseng usually refers to the root of Panax ginseng C.A. Meyer. Korean red ginseng (KRG) and fermented black ginseng (FBG) are most used processing varieties which are widely used in Northern China, Korea, and Japan.

Panax quinquefolium L. is also a perennial herb of Panax genus under Araliaceae family, also known as American ginseng, which is different medicinal plant from Panax ginseng C.A.Meyer. It is native to Canada's great Quebec and the United States' Wisconsin, and also planted in Beijing, and Changbai Mountain, China. As an imported traditional medicine, American ginseng is also widely used in China. 
Ginseng extract is very complex, there are many kinds of chemical components, and the components of different parts are different. Ginseng extract does not refer to chemical components, but mixtures. Generally, saponins, flavonoids and other chemical components can be extracted from ginseng. In traditional medicine, ginseng extract is widely used for its many beneficial effects, including the reversal of pathological and physiological changes induced by ischemia, stress, aging, and the alleviation of nausea and vomiting.

\section{Nausea and vomiting}

Ginseng extract is proved useful for chemotherapy-induced emesis both in ferret's and in rat's model respectively. A study using ferrets investigated the anti-emetic effect of KRG total extract on cisplatininduced nausea and vomiting. Intraperitoneal (i.p.) administration of cisplatin $(7.5 \mathrm{mg} / \mathrm{kg})$ induced both nausea and vomiting with one-hour latency. The episodes of nausea and vomiting reached a peak after $1.5 \mathrm{~h}$ and persisted for $3 \mathrm{~h}$. Treatment with KRG total extract via oral route significantly reduced the cisplatin-induced nausea and vomiting in a dose-dependent manner (with series doses of $0.3,1.0$, and $3.0 \mathrm{~g} / \mathrm{kg}$ of KRG total extract). Pretreatment with KRG total extract via oral route 1 and $2 \mathrm{~h}$ before cisplatin administration also significantly attenuated the cisplatin-induced nausea and vomiting. However, this did not occur with a pretreatment $4 \mathrm{~h}$ before cisplatin administration. These results were supportive of KRG total extract being utilized as an anti-emetic agent against nausea and vomiting caused by cisplatin chemotherapy [16]. The other similar study investigated the effect of KRG extract on cisplatin-induced pica in rat model. Rats were treated with extract before $(25,50$, and $100 \mathrm{mg} / \mathrm{kg})$ or after $(12.5,25$, and $50 \mathrm{mg} / \mathrm{kg})$ a single i.p. injection of cisplatin ( 7 and $6 \mathrm{mg} / \mathrm{kg}$, respectively). Extract pretreatment (25 and $50 \mathrm{mg} / \mathrm{kg}$ ) significantly attenuated cisplatininduced kaolin intake (at 24, 48, and $72 \mathrm{~h}$ ), and markedly improved intake of normal food at $48,72,96$, and $120 \mathrm{~h}$. Upon post-treatment of rats with extract $(12.5,25$, and $50 \mathrm{mg} / \mathrm{kg})$ at $24 \mathrm{~h}$, cisplatin-induced kaolin intake was markedly decreased, too. Notably, post-treatment with the lowest extract dose resulted in a significant anti-pica effect and improved food intake until $72 \mathrm{~h}$. The magnitude of body weight reduction was significantly diminished in rats pre-treated/post-treated with 25,50 , and $12.5 \mathrm{mg} / \mathrm{kg}$ extract. The anti-pica effects of extract were further confirmed with hematological and histopathological findings [17]. These two findings collectively indicated that ginseng extract has effect against emesis.

\section{Cachexia}

Cisplatin-induced sickness behaviors rat model was used to investigate the protective effect of ginseng extract on cachexia. Cisplatin was administered twice weekly (1-2 mg/kg, i.p.) for 5 consecutive weeks. Ginseng extract $(0,25$ and $50 \mathrm{mg} / \mathrm{kg}$, intragastric) was administered daily over the 5-week period of cisplatin exposure. Malaise, bodyweight and temperature, pain sensitivity, and endurance running were recorded at baseline and at five weekly intervals. The results showed that treatment with cisplatin produced severe signs of malaise, marked loss of bodyweight, hypothermia, hyperalgesia and reduction in running time. Co-treatment with ginseng extract completely prevented all cisplatin-induced alterations, indicating that treatment with ginseng extract exerted a protective effect in rat model of cachexia [18].

\section{Ototoxicity}

Cisplatin can induce ototoxicity, which represents irreversible sensorineural hearing loss and serious tinnitus in humans. A cell biology study investigated the protective effects of KRG extract on cisplatin-induced ototoxicity in auditory cells. Pretreatment with $2.5 \mathrm{mg} / \mathrm{mL}$ of KRG extract prior to application of $20 \mu \mathrm{M}$ of cisplatin significantly increased cell viability after $48 \mathrm{~h}$ of incubation in auditory cells. Pretreatment with ginseng extract significantly attenuated the cisplatin-induced increase in reactive oxygen species (ROS). KRG extract also inhibited the expression of caspase- 3 and poly-ADP-ribose polymerase related to cisplatin-induced apoptosis because a major mechanism of cisplatin-induced toxicity involves ROS production. Thus, KRG extract played both an anti-apoptotic and anti-oxidative role on cisplatin-induced ototoxicity in an auditory cell line [19].

\section{Nephrotoxicity}

Cisplatin-induced nephrotoxicity is a difficult issue in clinical practice, which occurs in $30 \%$ of all cisplatin treated patients seriously. Nephrotoxicity often limits its clinical implication, and may even cause patients to alter or give up cisplatin therapy. Ginseng is perhaps one of the most researched natural products to reduce cisplatininduced nephrotoxicity. A study utilized cisplatin-induced acute renal failure (ARF) model in Sprague Dawley rats to determine whether KRG extract can mitigate acute renal nephropathy. KRG extract was administered to rats by oral gavage once a day at doses of 100,300 , or $500 \mathrm{mg} / \mathrm{kg}$ for 28 days. On day 23, the animals received an i.p. injection of cisplatin $(5 \mathrm{mg} / \mathrm{kg})$ to induce ARF. Body weight gain, urine volume, BUN and creatinine concentrations, and expression of p53 were measured. Terminal deoxynucleotidyl transferase dUTP nick end-labeling was used to analyze apoptosis. Kidney tissues were analyzed by immunohistochemistry for inflammatory cytokines and histopathological examination. To identify the mechanism responsible for the Nephroprotective effects of KRG, malondialdehyde (MDA) concentration, the activities of the antioxidants superoxide dismutase (SOD) and GSH were measured. The results showed that KRG extract significantly decreased the levels of indicators of renal dysfunction, inflammatory cytokine expression, apoptosis, and MDA content in the kidney and significantly attenuated the histopathological changes associated with ARF. These findings suggested that KRG extract has Nephroprotective effects against cisplatin-induced ARF by reducing oxidative stress and inflammation [20].

It is worth mentioning that American ginseng leaves extract also showed similar nephron-protective effects. A mouse model of cisplatininduced acute kidney injury (AKI) was used. The levels of BUN and $\mathrm{SCr}$ were evidently increased in cisplatin-intoxicated mice, which were reversed by the leaves extract. Renal oxidative stress, evidenced by increased MDA level and decline of GSH and SOD activities, was significantly alleviated by the leaves extract pretreatment. The suppression of inflammatory response by the leaves extract was realized through the decrease the mRNA expression levels of tumor necrosis factor- $\alpha$ (TNF- $\alpha$ ) and interleukin- $1 \beta$ (IL-1 $\beta$ ) in kidney tissues. Furthermore, the leaves extract administration significantly suppressed the protein expression levels of nicotinamide adenine dinucleotide phosphate oxidase type 4 (Nox4), cleaved caspase-3, cleaved caspase-9, $\mathrm{Bax}$, nuclear factor- $\mathrm{KB}$ (NF- $\mathrm{B}$ ), cyclooxygenase-2 (COX-2), and inducible nitric oxide synthase (iNOS), suggesting the inhibition of apoptosis and inflammation response. Overall, the leaves extract may possess protective effects in cisplatin-induced AKI through suppression of oxidative stress, inflammation and apoptosis [21].

Besides, the processing variety of Panax ginseng, such as the fermented black ginseng (FBR), also showed similar results against cisplatin-induced nephrotoxicity in vivo and in vitro [22]. FBG is processed ginseng by the repeated heat treatment and fermentation 
of raw ginseng. Its protective effect against cisplatin-induced renal damage was tested in rats. FBG was orally administered every day at a dose of $150 \mathrm{mg} / \mathrm{kg}$ body weight for $10 \mathrm{~d}$, and a single dose of cisplatin was administered i.p. (7.5 $\mathrm{mg} / \mathrm{kg}$ body weight) with $0.9 \%$ saline on the 4th day. On the one hand, FBG displayed a strong 1,1-diphenyl-2-picrylhydrazyl (DPPH) radical-scavenging activity ( $50 \%$ inhibition concentration, $\mathrm{IC}_{50}=384 \mu \mathrm{g} / \mathrm{mL}$ ), which was mediated by the generation phenolic compounds. The decreased cell viability by cisplatin was recovered significantly after treatment with FBG in a dose-dependent manner. On the other hand, FBG displayed the protective effect on cisplatin-induced oxidative renal damage. The decreased creatinine clearance levels were reduced to the normal level after the administration of FBG. Moreover, FBG showed protective effects against cisplatin-induced oxidative renal damage in rats through the inhibition of NF- $\mathrm{kB} / \mathrm{p} 65$, COX-2, and caspase- 3 activation. These results collectively showed that FBG ameliorates the nephrotoxicity via regulating oxidative stress, inflammation, and apoptosis.

\section{Clinical research}

There are few clinical trials on cisplatin toxicity reversal by ginseng extract. Oncologists from China investigated the adjuvant therapeutic effects of ginseng extract on non-small cell lung cancer (NSCLC) patients treated with chemotherapy. A total of 60 patients with advanced NSCLC were divided into two groups using a random number table, i.e., the gemcitabine plus cisplatin (GP) chemotherapy alone group (26 patients) and the ginseng extract + GP chemotherapy group (34 patients), for 60-day treatment. Then patients were assessed according to the Fatigue Symptom Inventory (FSI), Chinese medicine (CM) symptoms score, Self-Rating Anxiety Scale, Self-Rating Depression Scale, Karnofsky Performance Status Scale, and Functional Assessment of Cancer Therapy-Lung. In addition, chemotherapy toxicity and tumor biomarkers were measured. Finally, for NSCLC patients after chemotherapy, ginseng extract significantly improved the FSI score, CM symptoms score, psychological status, physical conditions, and quality of life and reduced chemotherapy toxicity. However, the expression levels of carcinoembryonic antigen, cytokeratin-19 fragments, and neuron-specific enolase were not significantly different between the GP chemotherapy alone and the ginseng extract + GP chemotherapy groups or between pre- and post-treatments. This study demonstrated collectively that ginseng extract had an adjuvant effect on advanced NSCLC patients treated with chemotherapy [23].

\section{Mechanism}

One mechanism research on inflammatory response was conducted on the protective effect of American ginseng extract on cisplatininduced nephrotoxicity in mice. In the study, the histopathological changes and elevated levels of BUN and SCr caused by cisplatin were significantly diminished by American ginseng extract treatment. Oxidative stress caused by cisplatin, evidenced by increases in kidney tissues MDA content, renal 4-hydroxynonenal (4-HNE) levels and decreases of GSH and SOD contents, was significantly ameliorated by American ginseng extract pretreatment. The expression levels of tumor necrosis factor- $\alpha$ (TNF- $\alpha$ ), interleukin- $1 \beta$ (IL-1 $\beta$ ), cyclooxygenase- 2 (COX-2) and inducible nitric oxide synthase (iNOS) were inhibited by American ginseng extract treatment, suggesting a suppression of inflammatory response. Additionally, American ginseng extract clearly inhibited cisplatin-induced activations of nuclear factor-kappa B (NF$\kappa \mathrm{B})$ and mitogen activated protein kinase (MAPK) signal pathways. Supplementation of cisplatin-intoxicated mice with American ginseng extract also significantly reduced apoptotic protein levels of Bax, cleaved caspase- 3 , cytochrome $\mathrm{c}$ and increased anti-apoptotic protein $\mathrm{Bcl}-2$. These findings highlight nephron-protective effect of American ginseng extract against cisplatin-evoked nephrotoxicity through ROSmediated MAPK and NF- $\mathrm{KB}$ signaling pathways [24].

The chemical constituents derived from Panax ginseng extract are important material basis for mechanism research. Ginseng extract contains numerous phytochemicals such as ginsenoside, phenols and acidic polysaccharides that have significant pharmacological activities. Ginsenoside is unique triterpenoid saponins of the Panax plants and a group of the most potent biologically active components of ginseng. Identification of ginseng efficacy and in-depth mechanisms of action against cisplatin-induced toxicity have recently emerged as an interesting research area focusing on the kidney protective efficacy of ginseng. In-depth chemical components-efficacy researches have shown that treatment with ginsenosides (Rg3, Rb3, Rh2, Rk3, Rh4, Rd and Rg5), and pseudo ginsenoside (F11) ameliorate cisplatininduced nephrotoxicity in mice by reducing BUN and SCr [25]. Han et al investigated the kidney protective effect of FBG extract and its active component ginsenoside $\mathrm{Rg} 3$ against cisplatin-induced damage in pig kidney (LLC-PK1) cells. The results showed that the reduced cell viability induced by cisplatin was significantly recovered with FBG extract and ginsenoside Rg3 dose-dependently. The cisplatin-induced elevated protein levels of phosphorylated c-Jun N-terminal kinase (JNK), p53, and cleaved caspase-3 were decreased after co-treatment with FBG extract and ginsenoside Rg3. The elevated percentage of apoptotic LLC-PK1 cells induced by cisplatin treatment was significantly abrogated by co-treatment with FBG extract and ginsenoside Rg3. The data suggested that FBG extract and its major ginsenoside Rg3 might ameliorate cisplatin-induced nephrotoxicity in LLC-PK1 cells by blocking the JNK-p53-caspase-3 signaling cascade [26].

Another work evaluated the protective effects of ginsenoside $\mathrm{Rb} 3$ on cisplatin-induced renal damage and underlying mechanisms in vivo and in vitro, with cisplatin-induced ARF in ICR mouse model and HEK293 cell model. For animal experiment, renal function, kidney histology, inflammation, oxidative stress, relative protein molecules involved in apoptosis and autophagy signaling pathways were assessed. In addition, rapamycin (a specific inhibitor of mTOR), compound C (a specific inhibitor of AMPK) and acetylcysteine (NAC, a specific ROS scavenger) were employed to testify the effects of AMPK/mTOR signal pathway on the protective effects of Rb3 in HEK293 cells. The results showed that pre-treatment with $\mathrm{Rb} 3$ at doses of 10 and $20 \mathrm{mg} / \mathrm{kg}$ for ten days significantly reversed the increases in SCr, BUN and MDA, and decrease in GSH content and SOD activity. Histopathological examination further revealed that $\mathrm{Rb} 3$ inhibited cisplatin-induced nephrotoxicity. $\mathrm{Rb} 3$ diminished cisplatin-induced increase in protein expression levels of p62, Atg3, Atg5 and Atg7, and decrease in protein expression level of p-mTOR and the ratio of LC3-I/LC3-II, indicating that $\mathrm{Rb} 3$ suppressed cisplatin-induced activation of autophagy. Inhibition of autophagy induced inactivation of apoptosis, which suggested that autophagy played an adverse effect on cisplatin-evoked renal damage. Further, Rb3 might potentially modulate the expressions of AMPK-related signal pathways. These findings clearly suggested that Rb3-mediated alleviation of cisplatin-induced nephrotoxicity was in part due to regulation of AMPK-/mTOR-mediated autophagy and inhibition of apoptosis in vitro and in vivo [27].

Ginsenoside $\mathrm{Rh} 2$ (Rh2), same as $\mathrm{Rg} 3, \mathrm{Rb} 3$, has also received much attention due to a range of pharmacological actions. An in vivo mice model of DDP-induced nephrotoxicity was established by a single i.p. injection of DDP $(20 \mathrm{mg} / \mathrm{kg})$ to assess the effects of $\mathrm{Rh} 2$ on renal 
biochemical parameter, oxidative stress, inflammation tubular cell apoptosis and serum metabolic profiles. The results demonstrated that $\mathrm{Rh} 2$ protected against DDP-induced renal dysfunction and ameliorated DDP-induced oxidative stress, histopathological damage, inflammation and tubular cell apoptosis in kidney. Rh2 treatment had significantly increased expression of Bcl-2 and decreased expression of p53, Bax, cytochrome c, caspase-8, caspase- 9 , and caspase- 3 in kidney tissues. Metabolomic analysis identified 29 altered serum metabolites in $\mathrm{Rh} 2$ treatment mice. These results suggested that Rh2 protects against DDPinduced nephrotoxicity via action on caspase-mediated pathway [28].

The protective effect of ginsenosides $\mathrm{Rk} 3$ and $\mathrm{Rh} 4$ on kidney function was evaluated using in vitro and in vivo models of cisplatininduced acute renal failure. An enriched mixture of ginsenosides Rk3 and $\mathrm{Rh} 4$ was purified from heat- processed Panax ginseng. Cytotoxicity was induced by treatment of $20 \mu \mathrm{M}$ cisplatin to LLC-PK1 cells and rat model of acute renal failure was generated by single i.p. injection of $5 \mathrm{mg} / \mathrm{kg}$ cisplatin. Protective effects were assessed by determining cell viability, reactive oxygen species generation, blood urea nitrogen, serum creatinine, antioxidant enzyme activity, and histopathological examination. The in vitro assay results demonstrated that $\mathrm{Rk} 3-\mathrm{Rh} 4$ $(50 \mu \mathrm{g} / \mathrm{mL})$ significantly increased cell viability (4.6-fold), superoxide dismutase activity (2.8-fold), and glutathione reductase activity (1.5fold), but reduced reactive oxygen species generation (56\%) compared to cisplatin control cells. Rk3-Rh4 (6 mg/kg, per os) also significantly inhibited renal edema (87\% kidney index) and dysfunction $(71.4 \%$ blood urea nitrogen, $67.4 \%$ creatinine) compared to cisplatin control rats. Of note, RK3-Rh4 significantly recovered the kidney levels of catalase (1.2-fold) and superoxide dismutase (1.5-fold). The findings suggested that ginsenosides Rk3 and Rh4 protect the kidney from cisplatin-induced oxidative injury and help to recover renal function by restoring intrinsic antioxidant defenses [29].

\section{Ginseng reverse cisplatin resistance}

Multidrug resistance (MDR) is an obstacle for cancer chemotherapy. The clinical use of cisplatin has been limited by the frequent emergence of cisplatin-resistant cell populations and numerous other adverse effects. Currently, the reversal effect of natural products on cisplatin resistance in cancer chemotherapy has got attention. New agents are required to improve the therapy and health of cancer patients [30]. It is well known that ginseng extract exerts many beneficial effects, including the reversal of pathological and physiological changes induced by ischemia, stress, aging, and the alleviation of emesis. However, in this part of resistance reversal, ginseng extract constituents, instead of ginseng extract itself, is research hotspot.

Clinical drug resistance to platinum-based chemotherapy is considered a major impediment in the successful treatment of nonsmall-cell lung cancer (NSCLC). As early as in 2002, Zhang et al investigated the reversal effect of ginsenoside Rg3 on MDR and its mechanism utilizing human lung cancer cell lines A549 cells and A549/ DDP (cisplatin-resistance) cells [31]. MTT assays showed that Rg3 of low cytotoxicity concentration efficiently reverse MDR to cisplatin in human adenocarcinoma cell. The $\mathrm{IC}_{50}$ values of $\mathrm{Rg} 3$ on A549 and A549/DDP were 748 and $560 \mu \mathrm{mol} / \mathrm{L}$, respectively. At low-toxic dose $(10 \mu \mathrm{mol} / \mathrm{L}) \mathrm{Rg} 3$ effectively reversed A549/DDP resistance to cisplatin from $762.5 \mu \mathrm{mol} / \mathrm{L}$ to $103.8 \mu \mathrm{mol} / \mathrm{L}$ (about 7.3 fold) After A549/DDP cells were pretreated with $\operatorname{Rg} 3(10 \mu \mathrm{mol} / \mathrm{L})$ for $12,24,36$ and $48 \mathrm{~h}$, the sensitivity of cisplatin on A549/DPP cells were increased $0.95,1.58$, 7.58 and 10.38 fold respectively. The results suggest that the reversal of resistance by $\operatorname{Rg} 3$ was time-dependent which was via down-regulating
MDR1, MRP, and LRP mRNA and protein expression. Another same work used the same cell line, got the similar results, but focused on different mechanism. $\operatorname{Rg} 3(25 \mu \mathrm{g} / \mathrm{mL})$ reversed A549/DDP drugs resistance via decreasing cell membrane lipid fluidity and cell invasion ability, up-regulating metastasis-inhibiting gene nm23 and caspase-3 expression of A549/DDP cells [32] .

Programmed death ligand 1 (PD-L1) as one the most important immune checkpoint was verified to involve in chemotherapy resistance in non-small cell lung cancer (NSCLC). Rg3 is isolated from ginseng extract that is recognized to boost immune. A study was conducted to identify whether Rg3 could attenuate the PD-L1 expression induced by resistance to cisplatin and draw out the underlying mechanisms of PDL1 in this process in human A549 and A549/DDP cells. Cell viability was detected by MTT assay, the PD-L1, Akt and NF- $\mathrm{kB}$ p65 protein expression were detected using Western blot analysis, the $\mathrm{T}$ cells cytotoxicity to tumor cells was detected by crystal violet staining living residual tumor cells after co-culture of tumor cells and $\mathrm{T}$ cells. The results showed that $\operatorname{Rg} 3$ could inhibit the growth and alleviate the resistant to cisplatin of A549/DDP cells. PD-L1 was overexpression in A549/DDP cells than A549 cells. Rg3 could decrease the PD-L1 expression induced by chemo-resistance and resume the $\mathrm{T}$ cells cytotoxicity to cancer cells. NF- $\kappa$ B p65 and Akt were involved in the PD-L1 overexpression and restrained by $\mathrm{Rg} 3$. Therefore, $\operatorname{Rg} 3$ could be regarded as a new agent targeting PD-L1 in chemotherapy refractory NSCLC [33].

It was reported that 20(S)-ginsenoside $\mathrm{Rg} 3$ was able to regulate MDR in mouse leukemia cells. The present study investigated the effect of Rg3 on the MDR of A549 lung cancer cells. A cell viability assay revealed that $\mathrm{Rg} 3$ treatment increased cisplatin (DDP) cytotoxicity in DDP resistant A549 cells (A549/DDP). Furthermore, Rg3 increases the antitumor effect of DDP on A549/DDP xenograft mice. The expression of MDR-mediated proteins, including P-gp, MRP and lung resistance protein 1 (LRP1), was detected in tumor tissue of A549/DDP xenograft mice. The results revealed that $\operatorname{Rg} 3$ treatment inhibited the expression of these MDR-associated proteins. Additionally, 99mTc-MIBI singlephoton emission computed tomography was used to monitor the effect of Rg3 on cisplatin sensitivity of A549/DDP xenograft tumors. It was observed that uptake of $99 \mathrm{mTc}-\mathrm{MIBI}$ was increased by $\mathrm{Rg} 3$ treatment, which indicated that $\mathrm{Rg} 3$ is able to effectively enhance chemotherapy sensitivity of A549/DDP xenograft tumors. Taken together, these results revealed that $\mathrm{Rg} 3$ might be able to reverse MDR of lung cancer via the down-regulation of P-gp, MPR1 and LRP1v [34].

The nuclear factor erythroid 2-related factor 2 (NRF2) signaling pathway regulates the oxidative stress response, and in many cancer types, the high constitutive expression of NRF2 leads to proliferation and chemo-resistance. Ginsenoside Rd is the main active component of ginsenosides. Rd was found to inhibit the proliferation of A549 lung cancer cells and induce G0/G1 phase arrest. The $\mathrm{IC}_{50}$ of DDP, gemcitabine, and adriamycin were much higher in A549/DDP cells than in A549 cells. The A549/DDP cell lines developed MDR, accompanied by activation of MDR protein 1 and MRP, as well as NRF2 and its target genes. Treatment with Rd inhibited the NRF2 pathway and significantly sensitized A549/DDP cells to therapeutic drugs. In addition, NRF2 knockdown attenuated the synergistic effects of Rd in both A549 and A54/DDP cells. Taken together, these data showed that NRF2 plays an important role in acquired drug resistance in NSCLC, and Rd may ameliorate this chemo-resistance by downregulating the NRF2 pathway [35].

Rg3 also reversed cisplatin-resistant esophageal cancer cells. Cisplatin-resistant Eca109/DDP cells was used to investigate the effect 
and mechanism of $\mathrm{Rg} 3$. The results showed that ginsenoside $\operatorname{Rg} 3(2.5 \mu \mathrm{g} /$ $\mathrm{mL}$ ) can significantly reduce the $\mathrm{IC}_{50}$ of cisplatin on Eca109/DDP cells. Cells were arrested in G1 phase. Co-treatment of Rg3 and cisplatin significantly decrease the P-gp expression. Reduction of P-gp expression may be one of the mechanisms of the reversal effect of $\operatorname{Rg} 3[36,37]$.

The latest work found that ginsenoside $\mathrm{Rg} 3$ reverses cisplatin resistance of gastric cancer cells by down-regulation of PD-L1 [33]. Cisplatin-resistant gastric cancer cell line SGC7901/DDP was used. Rg3 could inhibit SGC7901 and SGC7901/DDP cell viability in dose and time-dependent manner. Pretreatment with low concentration of RG3 enhanced significantly the inhibition effect of DDP on SCG7901/ DDP cells proliferation $(\mathrm{p}<0.05)$. Pretreatment with low concentration of RG3 also decreased significantly the mRNA and protein expression of PD-L1 $(\mathrm{p}<0.05)$.

\section{Conclusion}

For few decades many achievement has been gained in ginseng extract research. Ginseng extract and chemical constituents exerted reversal of cancer resistance primary by reducing membrane transport proteins, decreasing tumor cell detoxication, repair, regulating signal transduction apoptosis genes, cell cycles, and etc. It is an interesting but confusing phenomena that why ginseng extract could prevent/ protect cisplatin-induced toxicity meanwhile its chemical constituent ginsenoside reverses cisplatin resistance. It might be possible due to the multi-components and multi-target effects through multipathway approach in all these natural products, as well their diverse pharmacological effects. However, inhibition on oxidative stress might be common for all these products. The underlying mechanism need be further studied in future. Lot of evidences suggests that ginseng extract may be a therapeutic promising tool for supportive care in oncology. Ginseng extract and its chemical constituents are more promising as potential candidates for addressing cisplatin resistance and it is necessary to carry out clinical studies to verify this efficacy. Although there are a few clinical researches, further studies with a larger sample size will confirm the current findings.

This study first summarized the interesting but seemingly confusing phenomena that ginseng extract could prevent/protect cisplatininduced toxicity meanwhile its chemical constituent ginsenoside reverses cisplatin resistance. Its potential molecular mechanism is worthy of further study.

\section{Funding}

This work was supported by Shanghai Municipal Clinical Pharmacy Key Specialty Construction Project (2018-2020) and Shanghai Science and Technology Commission Medical Guidance Project (19411971700).

\section{References}

1. Muggia F (2009) Platinum compounds 30 years after the introduction of cisplatin:implications for the treatment of ovarian cancer. Gynecol Oncol 112: 275281. [Crossref]

2. Xu F, Hu MY, Shi XH (1991) Urinary analysis of cisplatin by ultraviolet spectrophotometry. Journal of First Military Medical College 11: 33-332.

3. Liu GX, YangLF, ZhangBR, Xu F (2004) Time-dependent characteristics of the effect, drug adverse reaction and toxicity of cisplatin. Chinese Hospital Pharmacy Journal 24: 609-610. [Crossref]

4. Musthaba M, Baboota S, Athar TM, Thajudeen KY, Ahmed S, et al. (2010) Patented herbal formulations and their therapeutic applications. Recent Pat Drug Deliv Formul 4: 231-244. [Crossref]

5. Yin B, Fang DM, Zhou XL, Gao F (2019) Natural products as important tyrosine kinase inhibitors. Eur J Med Chem 182: 111664. [Crossref]
6. Wei HY, Yang Z, Xu F (2015) A minireview on the supportive care in cancer with traditional Chinese medicine. Int J Pharmacol 11: 222-225.

7. XuF, Zhen YS (2000) Biochemical modulation of EGCG in green tea in cancer chemotherapy. Foreign Medical Science Cancer Section 27: 100-102. [Crossref]

8. Cohen M, Hunter J (2017) Complementary medicine products: interpreting the evidence base. Intern Med J 47: 992-998. [Crossref]

9. Bhat ZY, Cadnapaphornchai P, Ginsburg K, Sivagnanam M, Chopra S, et al. (2015) Understanding the Risk Factors and Long-Term Consequences of Cisplatin-Associated Acute Kidney Injury: An Observational Cohort Study. PLoS One 10: Article ID e0142225. [Crossref]

10. Pabla N, Murphy RF, Liu K, Dong Z (2009) The copper transporter Ctr1 contributes to cisplatin uptake by renal tubular cells during cisplatin nephrotoxicity. Am J Physiol Renal Physiol 296: F505-511. [Crossref]

11. Yan M, Tang C, Ma Z, Huang S, Dong Z (2016) DNA damage response in nephrotoxic and ischemic kidney injury. Toxicol Appl Pharmacol 313: 104-108. [Crossref]

12. Di Pasqua AJ, Kerwood DJ, Shi Y, Goodisman J, Dabrowiak JC (2011) Stability of carboplatin and oxaliplatin in their infusion solutions is due to self-association. Dalton Trans 40:4821-4825. [Crossref]

13. Quintanilha JCF, Visacri MB, Sousa VM, Bastos LB, Vaz CO, et al. (2018) Cisplatininduced human peripheral blood mononuclear cells' oxidative stress and nephrotoxicity in head and neck cancer patients: the influence of hydrogen peroxide. Mol Cell Biochem 440: 139-145. [Crossref]

14. Xu F, Cai ZF (2004) Research progress on natural products for multidrug resistance in cancer. Chinese Journal Drug Application Monitoring 3: 57-60.

15. Qi ZL, Wang Z, Li W, Hou JG, Liu Y, et al. (2017) Nephroprotective Effects of Anthocyanin from the Fruits of Panax ginseng (GFA) on Cisplatin-Induced Acute Kidney Injury in Mice. Phytother Res 31: 1400-1409. [Crossref]

16. KimJH, Yoon IS, Lee BH, Choi SH, Lee JH, et al. (2005) Effects of Korean red ginseng extract on cisplatin-induced nausea and vomiting. Arch Pharma Res 28: 680-684. [Crossref]

17. RaghavendranHR, Rekha S, Shin JW, Kim HG, Wang JH, et al. (2011) Effects of Korean ginseng root extract on cisplatin-induced emesis in a rat-pica model. Food Chem Toxicol 49: 215-21. [Crossref]

18. Lobina C, Carai MA, Loi B, Gessa GL, Riva A, et al. (2014) Protective effect of Panax ginseng in cisplatin-induced cachexia in rats. Future Oncol 10: 1203-1214. [Crossref]

19. ImGJ, Chang JW, Choi J, Chae SW, Ko EJ, et al. (2010) Protective effect of Korean red ginseng extract on cisplatin ototoxicity in HEI-OC1 auditory cells. Phytother Res 24: 614-621. [Crossref]

20. KimYJ, Lee MY, Son HY, Park BK1, Ryu SY, et al. (2014) Red ginseng ameliorates acute cisplatin-induced nephropathy. Planta Med 80: 645-654. [Crossref]

21. Ma ZN, Li YZ, Li W, Yan XT4, Yang G, et al. (2017) Nephroprotective Effects of Saponins from Leaves of Panax quinquefolius against Cisplatin-Induced Acute Kidney Injury. Int J Mol Sci 18: E1407. [Crossref]

22. Jung K, An JM, Eom DW, Kang KS, Kim SN (2017) Preventive effect of fermented black ginseng against cisplatin-induced nephrotoxicity in rats. J Ginseng Res 41: 188194. [Crossref]

23. Jiang SL, Liu HJ, Liu ZC, Liu N, Liu R, et al. (2017) Adjuvant effects of fermented red ginseng extract on advanced non-small cell lung cancer patients treated with chemotherapy. Chin J Integr Med 23: 331-337. [Crossref]

24. Ma ZN, Liu Z, Wang Z1, Ren S1, Tang S1, et al. (2017) Supplementation of American ginseng berry extract mitigated cisplatin-evoked nephrotoxicity by suppressing ROSmediated activation of MAPK and NF-kB signaling pathways. Food Chem Toxicol 110: 62-73. [Crossref]

25. Li W, Yan MH, Liu Y, Liu Z, Wang Z, et al. (2016) Ginsenoside Rg5 Ameliorates Cisplatin-Induced Nephrotoxicity in Mice through Inhibition of Inflammation, Oxidative Stress, and Apoptosis. Nutrients 8: E566. [Crossref]

26. Han MS, Han IH, Lee D, An JM, Kim SN, et al. (2016) Beneficial effects of fermented black ginseng and its ginsenoside 20 (S)-Rg3 against cisplatin-induced nephrotoxicity in LLC-PK1 cells. J Ginseng Res 40: 135-140. [Crossref]

27. Xing JJ, Hou JG, Ma ZN, Wang Z, Ren S, et al. (2019) Ginsenoside Rb3 provides protective effects against cisplatin-induced nephrotoxicity via regulation of AMPK-/ mTOR-mediated autophagy and inhibition of apoptosis in vitro and in vivo. Cell Prolif 52: e12627. [Crossref] 
28. QiZ, Li W, Tan J, Wang C, Lin H, et al. (2019) Effect of ginsenoside Rh2 on renal apoptosis in cisplatin-induced nephrotoxicity in vivo. Phytomedicine 61: 152862. [Crossref]

29. Baek SH, Shin BK, Kim NJ, Chang SY, Park JH (2017) Protective effect of ginsenosides $\mathrm{Rk} 3$ and $\mathrm{Rh} 4$ on cisplatin-induced acute kidney injury in vitro and in vivo. $J$ Ginseng Res 41: 233-239. [Crossref]

30. Xu F, Cai ZF (2004) Antitumor resistance reversal agents from traditional Chinese medicine. Central South Pharmacy 2: 301-303.

31. Zhang W (2002) Reversal effect of ginsenoside Rg3 on cisplatin-resistant human lung adenocarcinoma cell line A549/DDP and its mechanism. Chinese Journal of Respiratory Critical Care Medicie 1: 100-103.

32. Wang Y, Liu JL, Liu L (2011) Studies of 20(R)-ginsenoside Rg3 on restraining metastasis and reversing multidrug resistance in A549/DDP cell line. Chinese Journal of Health Lab Technololgy 21: 609-614.
33. Jiang Z, Yang Y, Yang Y, Zhang Y, Yue Z, et al. (2017) Ginsenoside Rg3 attenuates cisplatin resistance in lung cancer by downregulating PD-L1 and resuming immune. Biomed Pharmacother 96: 378-383. [Crossref]

34. Liu C, Gong Q, Chen T, Lv J, Feng Z, et al. (2018) Treatment with 20(S)-ginsenoside $\mathrm{Rg} 3$ reverses multidrug resistance in A549/DDP xenograft tumors. Oncol Lett 15: 4376-4382. [Crossref]

35. Chian S, Zhao Y, Xu M, Yu X, Ke X, et al. (2019) Ginsenoside Rd reverses cisplatin resistance in non-small-cell lung cancer A549 cells by downregulating the nuclear factor erythroid 2-related factor 2 pathway. Anticancer Drugs 30: 838-845. [Crossref]

36. Li XP, Wang YS, Liu YW (2013) Reversing cisplatin-resistant of Eca109/DDP cell line by ginsenoside Rg3. Anatomy Research 35: 351-354.

37. Wan PW, Wang Q, Wan C (2018) Ginsenoside Rg3 reverses cisplatin resistance of gastric cancer cells by down-regulation of PD-L1. J Med Res 47: 120-125.

Copyright: (2019 Cai J. This is an open-access article distributed under the terms of the Creative Commons Attribution License, which permits unrestricted use, distribution, and reproduction in any medium, provided the original author and source are credited. 